\title{
Sound source reconstruction using inverse boundary element calculations
}

\author{
Schuhmacher, Andreas; Hald, Jørgen; Rasmussen, Karsten Bo; Hansen, Per Christian
}

Published in:

Acoustical Society of America. Journal

Link to article, DOI:

$10.1121 / 1.1529668$

Publication date:

2003

Document Version

Publisher's PDF, also known as Version of record

Link back to DTU Orbit

Citation (APA):

Schuhmacher, A., Hald, J., Rasmussen, K. B., \& Hansen, P. C. (2003). Sound source reconstruction using inverse boundary element calculations. Acoustical Society of America. Journal, 113(1), 114-127. https://doi.org/10.1121/1.1529668

\section{General rights}

Copyright and moral rights for the publications made accessible in the public portal are retained by the authors and/or other copyright owners and it is a condition of accessing publications that users recognise and abide by the legal requirements associated with these rights.

- Users may download and print one copy of any publication from the public portal for the purpose of private study or research.

- You may not further distribute the material or use it for any profit-making activity or commercial gain

- You may freely distribute the URL identifying the publication in the public portal 


\title{
Sound source reconstruction using inverse boundary element calculations
}

\author{
Andreas Schuhmacher ${ }^{\mathrm{a})}$ and Jørgen Hald \\ Brüel \& Kjær A/S, Skodsborgvej 307, DK-2850 Nærum, Denmark \\ Karsten Bo Rasmussen ${ }^{\text {b) }}$ \\ Ørsted.DTU, Section of Acoustic Technology, Building 352, Technical University of Denmark, \\ DK-2800 Kgs. Lyngby, Denmark \\ Per Christian Hansen \\ Informatics and Mathematical Modelling, Building 321, Technical University of Denmark, \\ DK-2800 Kgs. Lyngby, Denmark
}

(Received 8 February 2002; revised 28 September 2002; accepted 22 October 2002)

\begin{abstract}
Whereas standard boundary element calculations focus on the forward problem of computing the radiated acoustic field from a vibrating structure, the aim in this work is to reverse the process, i.e., to determine vibration from acoustic field data. This inverse problem is brought on a form suited for solution by means of an inverse boundary element method. Since the numerical treatment of the inverse source reconstruction results in a discrete ill-posed problem, regularization is imposed to avoid unstable solutions dominated by errors. In the present work the emphasis is on Tikhonov regularization and parameter-choice methods not requiring an error-norm estimate for choosing the right amount of regularization. Several parameter-choice strategies have been presented lately, but it still remains to be seen how well these can handle industrial applications with real measurement data. In the present work it is demonstrated that the L-curve criterion is robust with respect to the errors in a real measurement situation. In particular, it is shown that the L-curve criterion is superior to the more conventional generalized cross-validation (GCV) approach for the present tire noise studies. (C) 2003 Acoustical Society of America. [DOI: 10.1121/1.1529668]
\end{abstract}

PACS numbers: 43.20.Fn, 43.35.Sx, 43.60.Cg [MO]

\section{INTRODUCTION}

Our objective in this work is to compute the unknown surface velocity distribution on a complex acoustic source from measured acoustic field data. Hence, we are faced with an inverse source problem to be solved, which involves forming a transfer matrix relating the pressure at every field point to the normal component of the surface velocity. In the present work this forward modeling is done by means of a boundary element method (BEM). Several developments in the area of source reconstruction involving arbitrarily shaped geometries have been presented in the past. ${ }^{1}$ Earlier work presented the basic concept of using BEM (mainly the direct formulation) in reconstruction problems for setting up a transfer matrix, while the singular value decomposition (SVD) routine is part of the matrix inversion..$^{2-4}$ More recent work also deals with the reconstruction process based on indirect BEM for selecting optimum field points from a set of candidates. ${ }^{5}$ Applications involving inverse source problems based on BEM can be found, for example, within the automotive and aerospace industry. Both interior and exterior noise problems have been studied. ${ }^{6-9}$

Some parts of the sound field radiated by a vibrating structure die out very quickly away from the source and therefore contribute very little at the field microphone posi-

\footnotetext{
${ }^{a)}$ Electronic mail: schuhmache@bksv.com

${ }^{b}$ Present address: Oticon A/S, Strandvejen 58, DK-2900 Hellerup, Denmark.
}

tions; these sound field components are often referred to as the evanescent waves. The reconstruction of the particular vibration patterns that create the evanescent waves will involve a strong amplification of very small signal components, and as a consequence, the inverse problem is very sensitive to the noise and errors in the measured data. The inverse problem is therefore said to be ill-posed.

As a consequence of the inverse problem being illposed, the transfer matrix computed by the BEM is illconditioned, thus requiring special treatment. In that respect, the SVD is the preferred tool toward regularizing the illposed problem. Whereas setting up the transfer matrix and making use of its SVD is well documented, the final step of choosing the correct amount of regularization is not fully understood. The discrepancy principle, which uses a priori knowledge of the measurement errors, has been used in connection with Tikhonov regularization ${ }^{7}$ and Landweber iteration. ${ }^{10}$ The generalized cross-validation (GCV) method has also been used by several researchers in conjunction with Tikhonov regularization, and it has been shown to produce useful results for cases where spatially white noise contaminates the field data. ${ }^{11,12}$ Recent work compares several iterative and direct inverse methods for near-field acoustic holography, ${ }^{13}$ and among these we find a modified Tikhonov approach with the discrepancy principle and the GCV used as the parameter-choice method.

In the present work we describe an approach to the inverse problem based upon an indirect BEM formulation in 
conjunction with Tikhonov regularization, and using the SVD as an analysis tool. The influence of the regularization parameter and the filtering process associated with Tikhonov regularization is investigated by means of the L-curve, which is a $\log -\log$ plot of the solution's norm versus the norm of the residual vector. The L-curve, ${ }^{14}$ which is not used too often in connection with sound source reconstruction, seeks to determine the optimal amount of regularization, and this method is compared to the more popular GCV method. A practical case using a car tire and real test data demonstrates the performance of both parameter-choice methods. In addition, a comparison with planar near-field acoustic holography is made in order to compare the different source reconstructions.

Since the setup of the inverse acoustic source problem uses the BEM as a workhorse, some general boundary element issues will be discussed in Sec. II. In Sec. III we introduce inverse methods based on SVD and Tikhonov regularization, and different parameter-choice methods will be introduced. Implementation issues are discussed in Sec. IV, including how to set up the discrete ill-posed problem and bring it on Tikhonov form. In Sec. V we demonstrate the performance of the GCV and L-curve methods for a couple of inverse problems involving measurement data. Two different applications are studied; a stationary tire structure excited by a shaker and a tire rolling at constant speed.

\section{BOUNDARY INTEGRAL FORMULATIONS OF THE FORWARD PROBLEM}

An exterior acoustic forward problem is considered, that is, only the fluid outside a vibrating source surface having an arbitrary shape is addressed. No secondary sound sources or obstacles exist in the exterior region. The acoustic pressure in the fluid must satisfy the Helmholtz equation, and when subject to the Sommerfeld radiation condition and a Neumann boundary condition on the source surface, boundary integral formulations can be found. These express the acoustic pressure at field points in terms of the source surface velocity. Boundary integral equations provide a complete formulation of the acoustic problem, thus making these equations an obvious choice in connection with radiation and/or scattering problems involving arbitrarily shaped structures. Moreover, they have the advantage that the infinite nature of the exterior region is implicit in the surface representation.

The formulations are often classified as either direct or indirect-where the direct formulation corresponds to a Helmholtz integral equation and the numerous indirect formulations are based on layer potentials. The direct formulation is used more often, but it has some built-in limitations. A serious shortcoming is the failure when it is applied to bodies of thin shape or regular bodies with thin appendages. This thin-shape breakdown of the direct formulation was investigated extensively by Martinez ${ }^{15}$ and recently by Cutanda et al. ${ }^{16}$ and remedies ensuring a meaningful formulation for thin shapes were proposed.

In this work we focus on the indirect formulations, where the exterior acoustic field is expressed in terms of single layers, double layers, or combined single and double layers ${ }^{17-19}$ on the source boundary $S$. For a point $P$ lying strictly in the exterior region, we can express the pressure $p_{f}$ by the single-layer formulation,

$$
p_{f}(P)=-\int_{S} \sigma(Q) G(P, Q) d S(Q),
$$

where $\sigma(Q)$ is the source strength at $Q$ on $S$, and subscript $f$ denotes a field quantity. $G(P, Q)$ is the free-space Green's function satisfying the Sommerfeld radiation condition, i.e., $G(P, Q)=e^{-j k R} / 4 \pi R$, where $R$ is the distance between $P$ and $Q$, and $k$ is the acoustic wave number. This formulation can be viewed as a distribution of simple sources (monopoles) on the surface. The only remaining issue when solving the exterior Neumann problem is to determine the unknown source strengths $\sigma(Q)$ by matching them to the prescribed normal surface velocity $\nu_{n}$. While the surface pressure is a continuous function across $S$, the surface velocity $\nu_{n}$ exhibits a jump property and thus we can write the unknown source densities as

$$
\sigma(Q)=-j \omega \rho\left(\nu_{n}^{+}(Q)-\nu_{n}^{-}(Q)\right),
$$

where $\nu_{n}^{+}(Q)$ and $\nu_{n}^{-}(Q)$ are the velocities on the two sides of the surface at the point $Q$. Once the source strengths $\sigma(Q)$ are known, we are able to find the pressure on the surface or in the exterior by means of surface integration.

Another possibility is to express the field pressure by a double-layer formulation,

$$
p_{f}(P)=\int_{S} \mu(Q) \frac{\partial G(P, Q)}{\partial n_{Q}} d S(Q),
$$

where $\mu(Q)$ is the source density. This representation of the exterior field can be viewed as a distribution of double sources (dipoles) over $S$. In the double-layer representation, the source density represents a pressure jump across $S$ with a continuous surface velocity, and the density function $\mu$ is written for $P$ on $S$ as

$$
\mu(Q)=p_{s}^{+}(Q)-p_{s}^{-}(Q),
$$

where $p_{s}^{+}(Q)$ and $p_{s}^{-}(Q)$ are the pressures on the two sides of the surface at the point $Q$.

A third alternative is to make use of a combined approach by mixing the two layers, ending up with a general jump formulation that is able to model both a pressure jump and a surface velocity jump. This jump formulation is described in the literature as an alternative to formulations based on the Helmholtz integral equation, and it is applicable to thin structures frequently appearing in studies of real structures. ${ }^{20,21}$ We can consider the mixed layer formulation as based on vibrating thin shells with general impedance boundary conditions on both sides of the shells. ${ }^{22}$ The versatility of this general jump formulation, with the ability to handle complex structures, has led to the implementation in many commercial BEM codes, and it is used to study real industrial applications. Furthermore, this formulation is attractive because it can overcome the serious difficulty associated with irregular frequencies, where the equations fail to 
provide correct and meaningful results. However, the implementation is not as straightforward as one based on the Helmholtz integral equation.

In the present work we have chosen to work with a hard thin-shell formulation, i.e., the same normal velocity on each side of the surface. We thus end up with the double-layer formulation in Eq. (3). In order to establish the forward modeling problem, we must derive a complete procedure for computing the field pressure in the exterior region, given the normal surface velocities everywhere on the surface. This is done in two stages; first, the necessary "intermediate" source density $\mu$ must be determined from the known normal surface velocity, and then the acoustic field exterior to the surface is determined from straightforward integration. In this work we use a variational approach ${ }^{23}$ to provide the link between the surface parameters. This link between normal surface velocities and jump pressures is provided by considering the normal derivative of $p_{f}$ on the surface. This derivative, in turn, is equal to $-j \omega \rho \bar{\nu}_{n}(P)$, and we obtain

$$
-j \omega \rho \bar{\nu}_{n}(P)=\int_{S} \mu(Q) \frac{\partial^{2} G(P, Q)}{\partial n_{P} \partial n_{Q}} d S(Q),
$$

where the surface velocity $\bar{\nu}_{n}$ is known. Using the variational approach, the unknown pressure jump can be evaluated from $^{23}$

$$
\begin{aligned}
& -j \omega \rho \int_{S} \delta \mu(P) \bar{v}_{n}(P) d S(P) \\
& \quad=\int_{S} \int_{S} \delta \mu(P) \mu(Q) \frac{\partial^{2} G(P, Q)}{\partial n_{P} \partial n_{Q}} d S(P) d S(Q),
\end{aligned}
$$

where $\delta \mu(P)$ denotes a variation in the pressure jump. Here the hypersingularity associated with Green's function must be reduced to a weak singularity before the numerical integration.

Another problem occurring with BEM techniques in practice is the mathematical breakdown of the formulation at specific so-called critical or irregular frequencies. These special frequencies are related to eigenfrequencies of the interior region and will be present if an interior BEM problem is analyzed, but they will also affect the results of an exterior BEM analysis if no effort is made to remove their influence. Since meaningless results can occur at the irregular frequencies, we assume throughout this work that such frequencies are avoided. A modified formulation ${ }^{24}$ based on both jump terms, which incorporates impedance boundary conditions on the inner surface, can be used to avoid the problems with irregular frequencies; this feature is not included in the present work.

\section{THE INVERSE PROBLEM}

Whenever a sound source reconstruction problem is discretized-by means of BEM or other discretization methods - a so-called discrete ill-posed problem arises. In this section we describe how to deal with such problems.

The discretized problem can be written in general matrix-vector form as

$$
\mathbf{A x}=\mathbf{b},
$$

where $\mathbf{A}$ is a complex transfer matrix of dimension $m \times n$, i.e., $m$ rows and $n$ columns. Hence, the complex vectors $\mathbf{x}$ and $\mathbf{b}$ are of dimension $n$ and $m$, respectively. In the following we assume that the system is either square or overdetermined, i.e., $m \geqslant n$. The matrix $\mathbf{A}$ represents a model of the system under investigation while $\mathbf{b}$ is a data vector of known measured values. In our work the system under study is the radiation process from a source to a number of receivers, $\mathbf{b}$ represents the response at the position of the receivers, and the model represented by A should be "inverted" in order to deduce the source descriptors in $\mathbf{x}$. We will only consider errors $\mathbf{e}$ on the right-hand side, while errors in $\mathbf{A}$ are neglected. When Eq. (7) arises from discretization of an inverse problem, A will be very ill conditioned. Consequently, a straightforward solution of the discrete inverse problem is not satisfactory, and special regularization techniques should be employed in order to solve Eq. (7) in a nearby sense.

\section{A. Singular value decomposition}

Solving discrete ill-posed problems via a direct solution approach should preferably be combined with tools that provide some kind of analysis of the specific problem at hand. Such tools do not solve the problem itself, but will often provide the necessary information before any next step toward a useful solution of the problem is taken. When it comes to ill-conditioned matrices, the ultimate analysis tool seems to be the singular value decomposition (SVD). Given the transfer matrix $\mathbf{A}$, we can write the SVD of this matrix as $^{25}$

$$
\mathbf{A}=\mathbf{U} \mathbf{\Sigma} \mathbf{V}^{H}
$$

where $\mathbf{U}$ is a matrix of dimension $m \times n, \mathbf{V}$ is $n \times n$ and $\mathbf{\Sigma}$ is a diagonal matrix containing $n$ non-negative singular values $\sigma_{i}$ in nonincreasing order. The superscript $H$ in Eq. (8) denotes the complex conjugate transposed.

The two matrices $\mathbf{U}$ and $\mathbf{V}$ are written in the form

$$
\mathbf{U}=\left(\mathbf{u}_{1} \cdots \mathbf{u}_{n}\right), \quad \mathbf{V}=\left(\mathbf{v}_{1} \cdots \mathbf{v}_{n}\right),
$$

where $\mathbf{u}_{i}$ (dimension $m$ ) and $\mathbf{v}_{i}$ (dimension $n$ ) are called the left and right singular vectors. An important property of these vectors is their orthonormality, i.e., $\mathbf{U}^{H} \mathbf{U}=\mathbf{V}^{H} \mathbf{V}=\mathbf{I}_{n}$, with $\mathbf{I}_{n}$ the identity matrix of order $n$.

The condition number (based on the 2-norm) of $\mathbf{A}$ can be expressed as the ratio of the largest and the smallest singular value, ${ }^{25}$ i.e., $\operatorname{cond}(\mathbf{A})=\sigma_{1} / \sigma_{n}$. This quantity measures a solution's sensitivity to perturbations in the matrix itself as well as on the right-hand side. When we are dealing with ill-conditioned matrices, the range of singular values span many orders of magnitude and $\mathbf{A}$ is very ill conditioned. Some kind of filtering of the singular values must be added to the original problem before any solution step makes sense.

The behavior of the left and right singular vectors deserves special attention, because any filtering relies on this behavior. Hansen et al. ${ }^{26}$ demonstrate that the left and right singular vectors tend to have more oscillations as the index $i$ increases when the transfer matrix arises from discretization of an inverse problem. Thus, we will assume that highfrequency spatial information is linked to left- and rightsingular vectors having high index $i$. Likewise, the vectors 
with a small index represent slowly varying functions having few sign changes over the source surface. These considerations have not been proven, but for practical problems derived from integral equations they appear to be valid.

\section{B. Tikhonov regularization}

If all SVD components are taken into account, a very oscillating solution with a huge norm is obtained due to the error component $\mathbf{e}$ of the right-hand side $\mathbf{b}$. One way of suppressing the disastrous influence of these errors is to solve the initial ill-conditioned problem in a nearby sense with a constraint on the size of the sought solution. The solution size is linked to the solution smoothness, because ignoring components with a high index will lead to a smooth solution with reasonable size as measured by an appropriate norm.

In mathematical form, we can write this as a minimization problem, where the functional to be minimized involves a residual norm and a discrete smoothing norm $\|\mathbf{L x}\|_{2}$ acting on the solution vector:

$$
\underset{\mathbf{x}}{\min }\left\{\|\mathbf{A x}-\mathbf{b}\|_{2}^{2}+\lambda^{2}\|\mathbf{L} \mathbf{x}\|_{2}^{2}\right\}
$$

This form of regularization is known as Tikhonov regularization, which is discussed at length, e.g., in Hansen. ${ }^{27}$ The regularization parameter $\lambda$ is the only input when a suitable smoothing norm has been defined. A possible implementation is described in Sec. IV B. If $\lambda=0$ we obtain the leastsquares problem and the unregularized solution is computed. A large $\lambda$ favors a small solution size at the cost of a large residual norm. Hence, $\lambda$ controls the degree to which the sought regularized solution should fit to the measured data in b or have a small solution size. Clearly, solving Eq. (10) involves a trade-off between the residual norm and the solution size, and this is determined by the single parameter $\lambda$. We return to our choice of $\mathbf{L}$ later.

For the choice of $\mathbf{L}=\mathbf{I}_{n}$, the Tikhonov regularized solution can be written conveniently in terms of the SVD of $\mathbf{A}$ $\operatorname{as}^{27}$

$$
\mathbf{x}_{\lambda}=\sum_{i=1}^{n} \frac{\sigma_{i}^{2}}{\sigma_{i}^{2}+\lambda^{2}} \frac{\mathbf{u}_{i}^{H} \mathbf{b}}{\sigma_{i}} \mathbf{v}_{i} .
$$

The filtering is described by the filter factors $f_{i}=\sigma_{i}^{2} /\left(\sigma_{i}^{2}\right.$ $\left.+\lambda^{2}\right)$. In practice, the regularization parameter is chosen to lie between the highest and smallest singular value. Hence, the filter gradually dampens singular values smaller than $\lambda$.

\section{Parameter-choice methods}

The main difficulty with regularization methods is that a proper choice of the amount of regularization is not easily found. Choosing the continuous parameter $\lambda$ in Tikhonov regularization is not straightforward. Ideally, we would like to determine the optimal regularization parameter such that the computed regularized solution provides the best possible estimate of the exact solution. The difficulty is that the exact solution is not available. Regularization parameters that are good approximations to the optimal ones can, however, be estimated by means of parameter-choice methods. Williams ${ }^{13}$

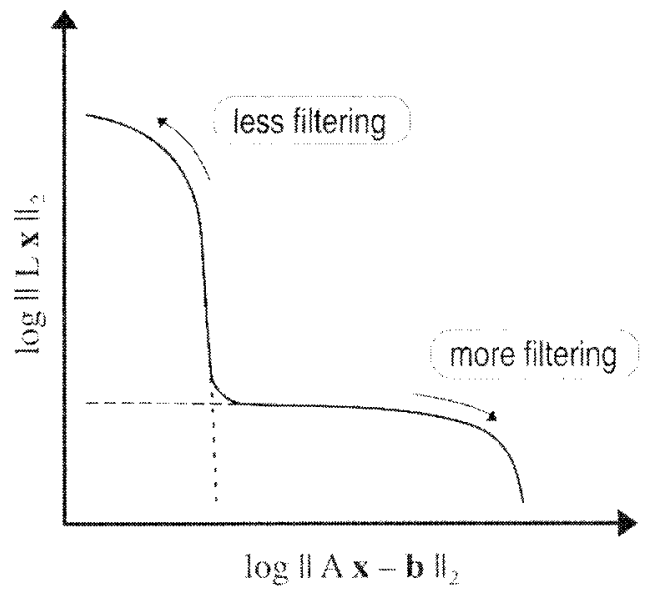

FIG. 1. The generic L-curve form; a plot in the log-log scale of the discrete smoothing norm versus the residual norm as a function of regularization parameter $\lambda$. From Ref. 27.

used the discrepancy principle and its compensated version, which require estimating the error norm $\|\mathbf{e}\|_{2}$ to determine the regularization parameter. The error norm can be estimated from the measurement data using the left-singular vectors, however, a very good estimate of the error norm is usually needed in order for the discrepancy principle to be robust, cf. Sec. 7.7 in Ref. 27.

Hence, there is a need for parameter-choice methods that do not require information about the error norm $\|\mathbf{e}\|_{2}$. Here we consider two $\|\mathbf{e}\|_{2}$-free methods for extracting an optimal regularization parameter. The first method, which is very popular, is called generalized cross-validation (GCV). It is based on the philosophy that an arbitrary element $b_{i}$ of the right-hand side should be predicted well by the regularized solution, when this element is left out of the inverse problem. This method works well if the noise is spatially white, i.e., if the elements of the noise vector are unbiased and have the same variance. Useful reconstructions were obtained by Nelson ${ }^{11,12}$ for a couple of inverse source problems with random errors representing measurement noise and using, among other techniques, the GCV as a parameter-choice method.

Another $\|\mathbf{e}\|_{2}$-free approach to estimating the regularization parameter in Tikhonov regularization is the so-called L-curve criterion. ${ }^{14}$ The idea behind the L-curve is to plot the discrete smoothing norm of the regularized solution versus the residual norm in $\log -\log$ scale, for all valid regularization parameters. The generic form of the L-curve is plotted in Fig. 1. The smoothing norm here is $\left\|\mathbf{L x}_{\lambda}\right\|_{2}$ and the residual norm is $\left\|\mathbf{A} \mathbf{x}_{\lambda}-\mathbf{b}\right\|_{2}$, i.e., the two terms occurring in the functional to be minimized in Tikhonov regularization. The shape of the L curve can be explained by considering its different sections. The horizontal part of the curve is characterized by solutions that have been smoothed too much (overregularized), whereas the vertical part is characterized by solutions dominated by the effects of errors (underregularized). In between these parts, we find solutions representing a balance between fitting the solution to data and keeping the solution's smoothing norm small. The optimum 
value of the regularization parameter defined by the L-curve criterion is at the corner of the curve, where we find a solution that has a reasonably small residual as well as a reasonably limited smoothing norm. We note that this is only possible in a plot in $\log -\log$ scale. ${ }^{28}$ A number of simulated case studies $^{29}$ dealing with simulated noise/error sources revealed that the L-curve criterion is more robust than the GCV method, especially when the added noise is non-white. This is an important finding before studying real applications, where the noise component of the field data cannot be considered to be white.

\section{IMPLEMENTATION OF THE INVERSE SOURCE PROBLEM}

Integral formulations are usually implemented numerically by a boundary element method where the surface of the boundary is replaced by a number of interconnected elements. These surface elements can typically be either triangles or squares, and the associated interpolation functions for the source values are typically linear or quadratic. In the present study triangular linear elements are used for describing the geometry as well as the acoustic variables on the surface. Hence, linear interpolation is used throughout for the geometry as well as the acoustic parameters, and the elements are therefore isoparametric, i.e., the same order of shape function is used in both cases. We note the general rule of thumb that the element size should not exceed one-fourth of the shortest wavelength for the linear elements.

First we derive the set of matrix equations needed to formulate the inverse problem, and we define the transfer matrix associated with the problem. The matrices are easily computed by means of the developed indirect BEM implementation. Subsequently, we will establish a functional amenable to Tikhonov regularization that will be the type of regularization imposed on the problem. Since the modeling of the inverse problem is linked to the BEM in acoustics, we will use the term Inverse Boundary Element Method (IBEM) or inverse BEM.

\section{A. Formation of the inverse BEM transfer matrix}

We will consider the surface $S$ of a closed or open structure, and normal vector on the surface $S$ points into the exterior. We assume that $m$ microphones are situated in the exterior region of the source. Basically these microphones can be located anywhere in this region, but some locations may be preferred over others. ${ }^{5,32}$ For every microphone position we will then measure an acoustic pressure.

The goal of the discretization of Eqs. (3) and (6) is to link the $m$ measured field pressures to an acoustic quantity on the source surface. From the indirect formulation we first establish the relation between the field pressures $p_{f}$ and the pressure jump $\mu$ on the source surface by discretization of the integral representation, Eq. (3). For each boundary element we introduce the vectors $\mathbf{N}_{i}$ and $\boldsymbol{\mu}_{i}$, which represent the shape function for linear triangles and the nodal values of the pressure jump distribution, respectively. This leads to the discretized problem

$$
p_{f}(P)=\sum_{i=1}^{e} \int_{S_{i}} \mathbf{N}_{i}^{T}(Q) \boldsymbol{\mu}_{i} \frac{\partial G(P, Q)}{\partial n_{Q}} d S_{i}(Q),
$$

where $e$ is the number of boundary elements.

For each measurement point we now use Eq. (12) to express the measured field $p_{f}$ in terms of the nodal values of the pressure jumps. The integrals in Eq. (12) can be evaluated using standard Gauss quadrature, as long as the point $P$ is not too close to the source surface. Otherwise, singularities involved in the integral formulation should be removed for $P$ in the very close near-field. A modified boundary element formulation based on Helmholtz integral equation has been reported to overcome such problems..$^{30,31}$ In our case the minimum distance between field points and source surface for BEM calculations is set to one-half an element length. Expressed in matrix-vector form, the discretized problem becomes

$$
\mathbf{p}_{f}=\mathbf{G} \boldsymbol{\mu},
$$

where $\mathbf{p}_{f}$ is a complex vector containing the $m$ field pressure values at a specific frequency, and $\boldsymbol{\mu}$ is a vector with the pressure jump values on the discretized surface containing $n$ nodes. The $m \times n$ transfer matrix $\mathbf{G}$ is notoriously ill conditioned.

Rather than solving Eq. (13) for $\boldsymbol{\mu}$ given a known vector $\mathbf{p}_{f}$, we will relate the field pressure vector to the normal surface velocity vector $\mathbf{v}_{n}$. This is done by means of the relation between pressure jump and normal surface velocity from the indirect boundary element formulation Eq. (6) which, when discretized, takes the form

$$
\begin{aligned}
&-j \omega \rho \boldsymbol{\delta} \boldsymbol{\mu}^{T}\left(\int_{S} \mathbf{N} \cdot \mathbf{N}^{T} d S(P)\right) \mathbf{v}_{n} \\
& \quad=\boldsymbol{\delta} \boldsymbol{\mu}^{T}\left(\int_{S} \int_{S} \mathbf{N} \frac{\partial^{2} G(P, Q)}{\partial n_{P} \partial n_{Q}} \mathbf{N}^{T} d S(P) d S(Q)\right) \boldsymbol{\mu},
\end{aligned}
$$

or in matrix-vector form,

$$
-j \omega \rho \mathbf{B} \mathbf{v}_{n}=\mathbf{Q} \boldsymbol{\mu} .
$$

The two matrices $\mathbf{B}$ and $\mathbf{Q}$ are symmetric, i.e., $\mathbf{B}=\mathbf{B}^{T}$ and $\mathbf{Q}=\mathbf{Q}^{T}$.

Combining Eqs. (13) and (15) we then obtain

$$
\mathbf{p}_{f}=-j \omega \rho \mathbf{G} \mathbf{Q}^{-1} \mathbf{B} \mathbf{v}_{n}=\mathbf{H} \mathbf{v}_{n} .
$$

Here, we have introduced the $m \times n$ rectangular transfer matrix, $\mathbf{H}=-j \omega \rho \mathbf{G} \mathbf{Q}^{-1} \mathbf{B}$, which is further analyzed in our IBEM problems. A similar way of assembling a transfer matrix using indirect BEM has been done by Zhang et al. ${ }^{5,32}$

Since $\mathbf{G}$ is ill-conditioned for measurement points lying strictly in the exterior, this property is carried over to the transfer matrix $\mathbf{H}$ that relates the normal surface velocity at nodes to acoustic pressure at measurement points. Note that the assembly of $\mathbf{H}$ necessitates the inverse of $\mathbf{Q}$, which will not cause trouble as long we avoid irregular frequencies (interior Neumann eigenfrequencies) for a closed structure. Consequently, Eq. (16) represents a discrete ill-posed problem of the form studied in Sec. III. Treating irregular frequencies in inverse BEM were discussed in Ref. 32 based on 
the same considerations as in Sec. II. How to solve Eq. (16) in the nearby sense by formulating a Tikhonov problem is discussed next.

\section{B. Tikhonov problem}

The regularization imposed in this investigation is based on Tikhonov's method with a parameter-choice method to estimate the optimal regularization parameter. In general form, the minimisation problem for Tikhonov regularization is written as

$$
\min _{\mathbf{v}_{n}}\left\{\left\|\mathbf{H v}_{n}-\mathbf{p}_{f}\right\|_{2}^{2}+\lambda^{2}\left\|\mathbf{L} \mathbf{v}_{n}\right\|_{2}^{2}\right\} .
$$

The discrete smoothing norm $\left\|\mathbf{L} \mathbf{v}_{n}\right\|_{2}$ must be chosen to preferably reflect some physical quantity to be penalized, that is, to have limited size. One such quantity could be some type of energy associated with a vibrating thin shell since the model is based upon shells. The equivalent kinetic energy of the entire shell structure makes sense, since the size of this quantity will increase dramatically if the nonradiating components have too large an influence on the regularized solution.

The "kinetic energy smoothing norm" is derived by considering the squared surface velocity over the shell surface. Since we only have access to the normal component of surface velocity, the equivalent total kinetic shell energy is written as

$$
\int_{S}\left|\nu_{n}\right|^{2} d S=\int_{S} \nu_{n} \nu_{n}^{*} d S,
$$

where the asterisk denotes a "complex conjugate." We can discretize this quantity using standard Gaussian quadrature and the discretization of Eq. (18) is written as

$$
\int_{S} \mathbf{v}_{n}^{H} \mathbf{N N}^{T} \mathbf{v}_{n} d S=\mathbf{v}_{n}^{H} \mathbf{B} \mathbf{v}_{n},
$$

with the shape function vector $\mathbf{N}$, and $\mathbf{B}$ is similar to the one defined via Eq. (14).

To simplify the calculations in the Tikhonov regularization, we may approximate the total surface function $\nu_{n} \nu_{n}^{*}$ as a linear function. Consequently, we end up with a diagonal matrix $\mathbf{D}$ that can be considered as an approximation to $\mathbf{B}$. Using this approach, the surface integral is written as

$$
\mathbf{v}_{n}^{H}\left(\int_{S} \operatorname{diag}(N) d S\right) \mathbf{v}_{n}=\mathbf{v}_{N}^{H} \mathbf{D} \mathbf{v}_{n} .
$$

Here the integrand is a sum of element areas weighted by the shape functions. Hence, only one Gauss point for each element integration is required. For each element integration over $S_{i}$, the value $\frac{1}{3} S_{i}$ is added on the diagonal of the area matrix for each node participating in element $i$. As a result, the diagonal element of $\mathbf{D}$ corresponding to a node in the BEM model will contain one-third of the total area of all elements of which the node takes part. Thus, the kinetic energy smoothing norm simply introduces an area weighting of the nodal values. The discrete smoothing norm is now written as

$$
\mathbf{v}_{n}^{H} \mathbf{D} \mathbf{v}_{n}=\left\|\mathbf{D}^{1 / 2} \mathbf{v}_{n}\right\|_{2}^{2},
$$

where the regularization matrix $\mathbf{D}^{1 / 2}$ is found by taking the square root of all the diagonal entries. Had our matrix not been diagonal, Cholesky factorization would have been necessary. Notice that if the element size is almost constant over the entire mesh surface, $\mathbf{D}$ will be close to the identity matrix, except for a multiplication constant. In that case, the standard form ( $\left.\mathbf{L}=\mathbf{I}_{n}\right)$ of Eq. (17) could just as well be used. However, for a general mesh $\mathbf{D}^{1 / 2}$ should be used instead of the identity matrix. Hence, the general form Tikhonov regularization is rewritten in standard form and solved. ${ }^{29}$

\section{CASE STUDIES}

In order to demonstrate the capabilities of inverse BEM, some real-life applications have been studied. ${ }^{29}$ The sound sources considered and modeled are based on a real tire structure mounted on a rim and loaded onto a drum surface. The tire is a standard tire for passenger cars and its characteristics as a source were studied as a stationary tire excited by a shaker as well as a rolling tire with a constant speed of $80 \mathrm{~km} / \mathrm{h}$ on the dynamometer.

\section{A. Tire measurements}

The measurements were recorded using a planar microphone array for the side measurement, and a curved array for close-up measurements at the front and the rear of the tire. Moreover, four reference microphones were distributed around the tire in order to allow the use of a scan technique and to derive a principal component description of the sound field. In this way, the problem of limited coherence between individual sources is addressed. Two of these four reference microphones were placed on the ground in front of and behind the tire, while the other two microphones were located in the air above the tire. When dealing with cross-spectral holography techniques and sound radiation from tires, four reference microphones seem to be sufficient. ${ }^{33}$ For the tire side, a $6 \times 6$ microphone array was scanned across a plane area with dimensions $1.7 \times 0.9 \mathrm{~m}$. The microphone spacing in the horizontal and vertical direction was $0.05 \mathrm{~m}$, giving a total of 665 measurement points in the plane, which was located $0.07 \mathrm{~m}$ from the sidewall of the tire. In addition, a curved array of 12 microphones scanned the rear and front of the tire. The curved array was scanned across the curved surface in 13 steps with a step size of $0.05 \mathrm{~m}$, thus giving 156 measurement points. The distance between the curved array and the curved part of the tire was about $0.07 \mathrm{~m}$. The front of the tire was not measured during the standing tire measurement, as the shaker occupied part of the space there. In total, 821 measurement positions were made for the shaken tire and 977 for the rolling tire. In addition to the IBEM reconstructions, we processed the data recorded at the tire side using Near-field Acoustic Holography as implemented in the Brüel \& Kjær STSF ${ }^{34,35}$ system. This allows for a comparison of the transformed sound fields obtained using IBEM and STSF. For example, we can compare the sound field reconstructions close to the tire side obtained by the two methods. 
In order to model the acoustic environment, a boundary element mesh of the complete tire was made and the hard ground is taken into account via an infinite rigid plane located $0.05 \mathrm{~m}$ below the tire. The tire boundary element mesh consists of linear triangles of maximum length $0.05 \mathrm{~m}$, resulting in 1052 elements and 526 nodes. The tire mesh including meshes of the side measurement and the rear measurement is shown in Fig. 2.

\section{B. Stationary tire excited by a shaker}

First, we study the inverse BEM problem for the shaken tire at a low frequency, $100 \mathrm{~Hz}$, the cross-spectral measurement being made with a frequency bandwidth equal to 20 Hz. The field pressure information from the measured 821 points close to the tire surface will now be used to establish an inverse problem, where the normal surface velocity at the 526 boundary nodes is sought. The transfer matrix is thus overdetermined and of size $821 \times 526$. As a result of the principal component description of the sound field, we can consider each principal hologram as a field pressure distribution and we can process the holograms individually, since they are mutually independent (incoherent). That is, for each principal hologram we determine a regularized surface velocity distribution by means of the SVD and Tikhonov regularization, where the optimum regularization parameter is chosen by either GCV or the L-curve criterion. The total surface velocity level is obtained by summing the principal component contributions on a power basis, subject to a Wiener filter
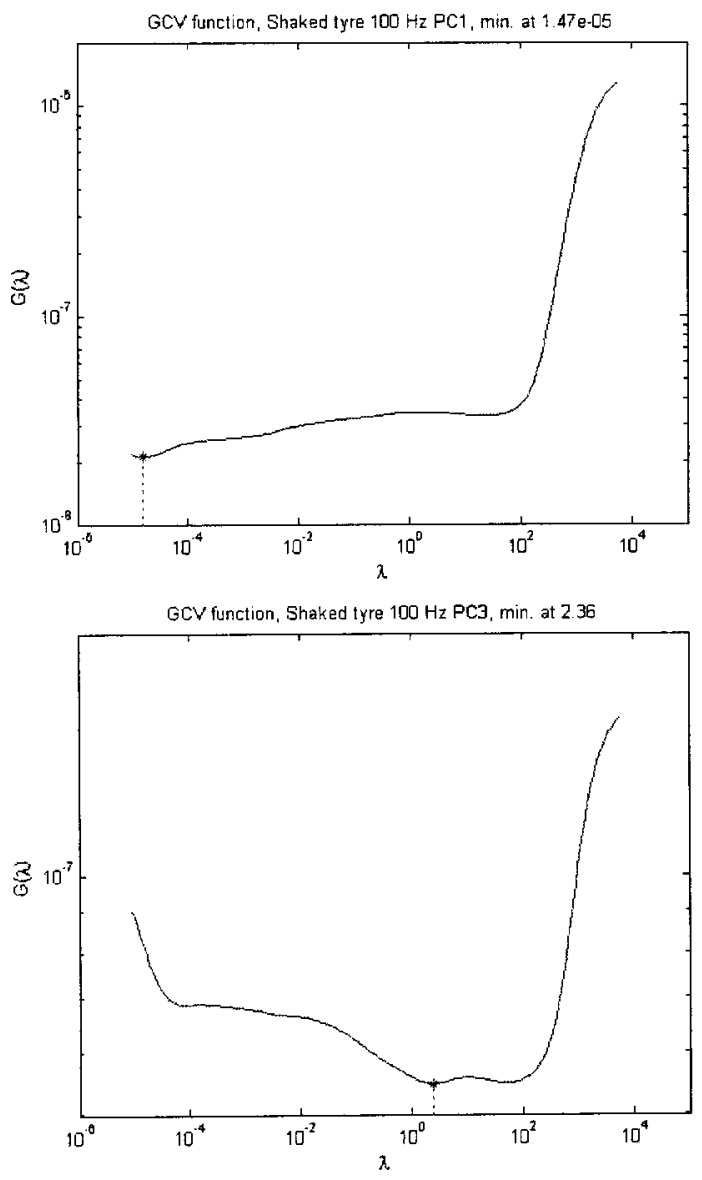

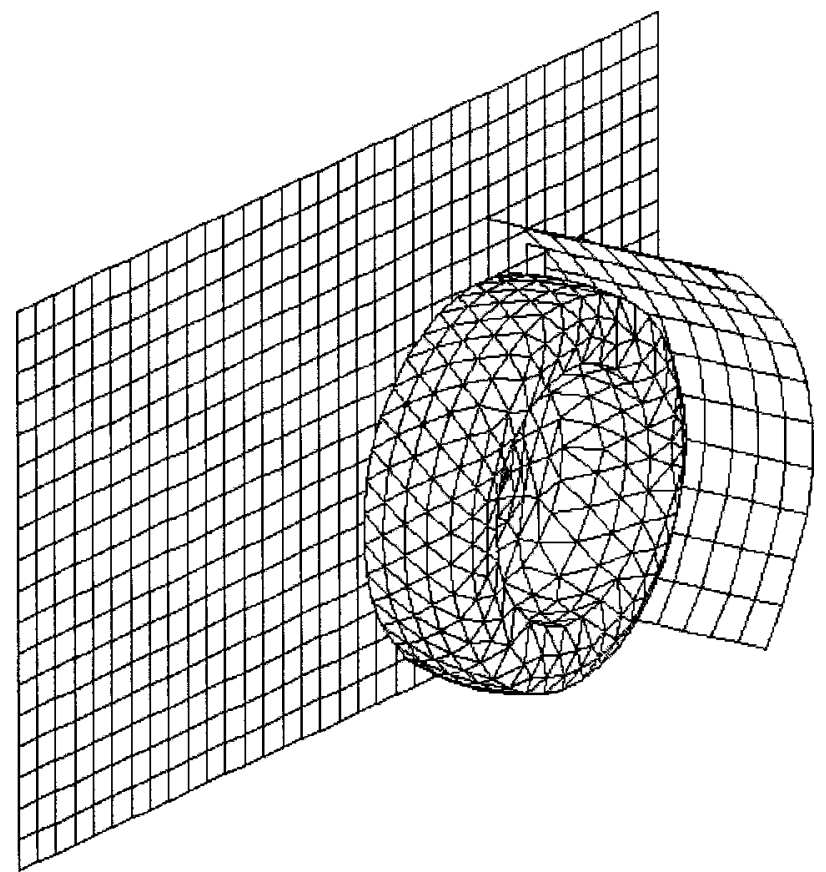

FIG. 2. Tire mesh (1052 elements, 526 nodes) and field point mesh (821 points) for stationary tire application.

controlled by the principal autopowers. In our case the filter has a dynamic range of $15 \mathrm{~dB}$ with a smooth cutoff, which means that the first and the second principal component are unaffected by the filter, whereas the third and the fourth prin-
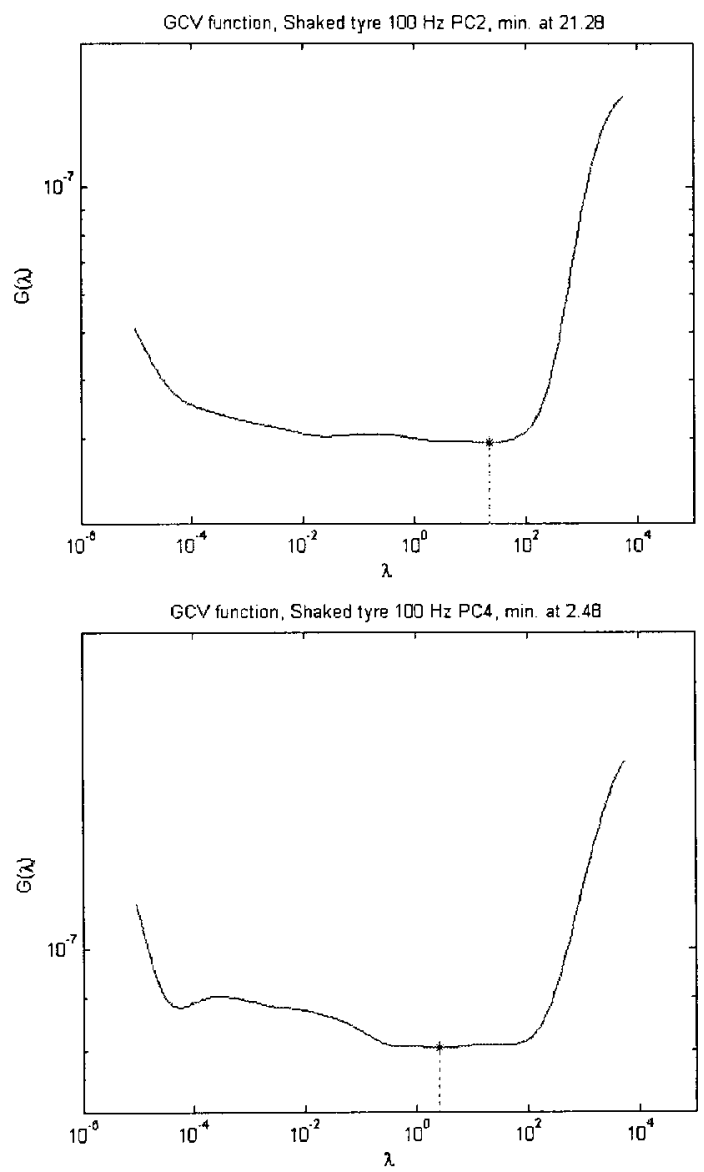

FIG. 3. GCV functions processed for each principal hologram for shaker excited tire at $100 \mathrm{~Hz}$. 

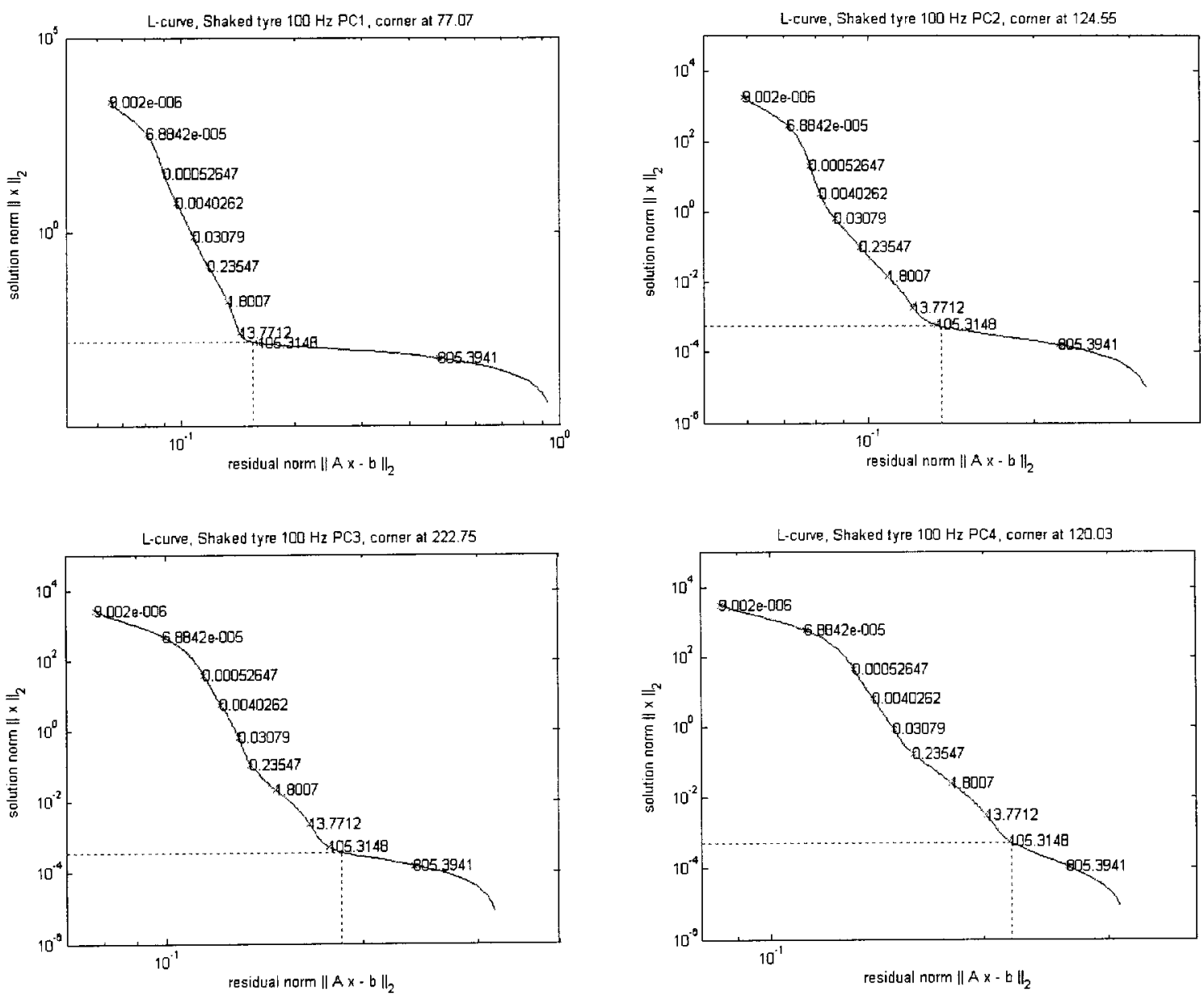

FIG. 4. L-curves corresponding to each principal hologram for a shaker excited tire at $100 \mathrm{~Hz}$. The regularization parameter is marked on the curve.

cipal component are attenuated. In the chosen frequency band, the autopowers of the second, third, and fourth principal component were 10,16, and $19 \mathrm{~dB}$, respectively, below the autopower of the first principal component. Since there is only one source mechanism (the shaker), one would expect only a single dominating principal component. A major reason for this not happening is the rather large measurement bandwidth. If within the measurement bandwidth the source vibration pattern changes a lot as a function of frequency, the different patterns will be seen to some degree as independent sources. This could explain the existence of the principal component, which is only $10 \mathrm{~dB}$ below the highest one. Other reasons for nonzero principal components are finiteaveraging time, nonstationarity and nonlinear dynamic behavior of structural components.

An inspection of the SVD components (not shown here) reveals that a regularization parameter $\lambda$ of the order 100 is appropriate for each principal component. First the GCV method was used to determine the optimum $\lambda$ for each principal component, and the GCV functions are shown in Fig. 3. It is clear that the estimated regularization parameters are too small, thus resulting in undersmoothed solutions completely dominated by measurement errors. Hence, the regularized surface velocity solution obtained by this method will be meaningless. This is surprising in the sense that the stationary tire excited by a shaker is the simpler one of the two applications. The probable cause is that the measurement er- rors are not spatially white, as assumed by the GCV method. We mention two error types.

(1) Transducer mismatch. These errors "follow" the array transducers during the scanning, and the resulting errors in the measured sound pressure will therefore be correlated with the true sound pressure.

(2) Transducer position errors. The measurement positions are never exactly known in the coordinate system of the BEM model. The resulting errors in the measured pressure will also tend to be correlated with the true pressure.

Further errors may occur as a result of the drum and as a result of a large obstacle located close to the tire side, which was not scanned. This obstacle was mounted to the rim of the tire but just like the drum it is not part of the acoustic boundary element model for the inverse analysis.

When the same problem is analyzed using the L-curve, we get the four curves shown in Fig. 4. Especially the first Lcurve exhibits the generic shape of Fig. 1 with a well-defined corner. Furthermore, the computed regularization parameters are all close to 100, which agrees very well with our SVD analysis. The dynamic range of the applied Tikhonov filters, i.e., $20 \log \left(\sigma_{1} / \lambda_{\mathrm{L}}\right)$, is $37,33,28$ and $33 \mathrm{~dB}$ for principal component one to four, respectively; here $\lambda_{\mathrm{L}}$ is the optimum regularization parameter computed by the L-curve criterion, and $\sigma_{1}$ is the largest singular value. The solutions obtained by the GCV are seen to be located on the upper of the ver- 

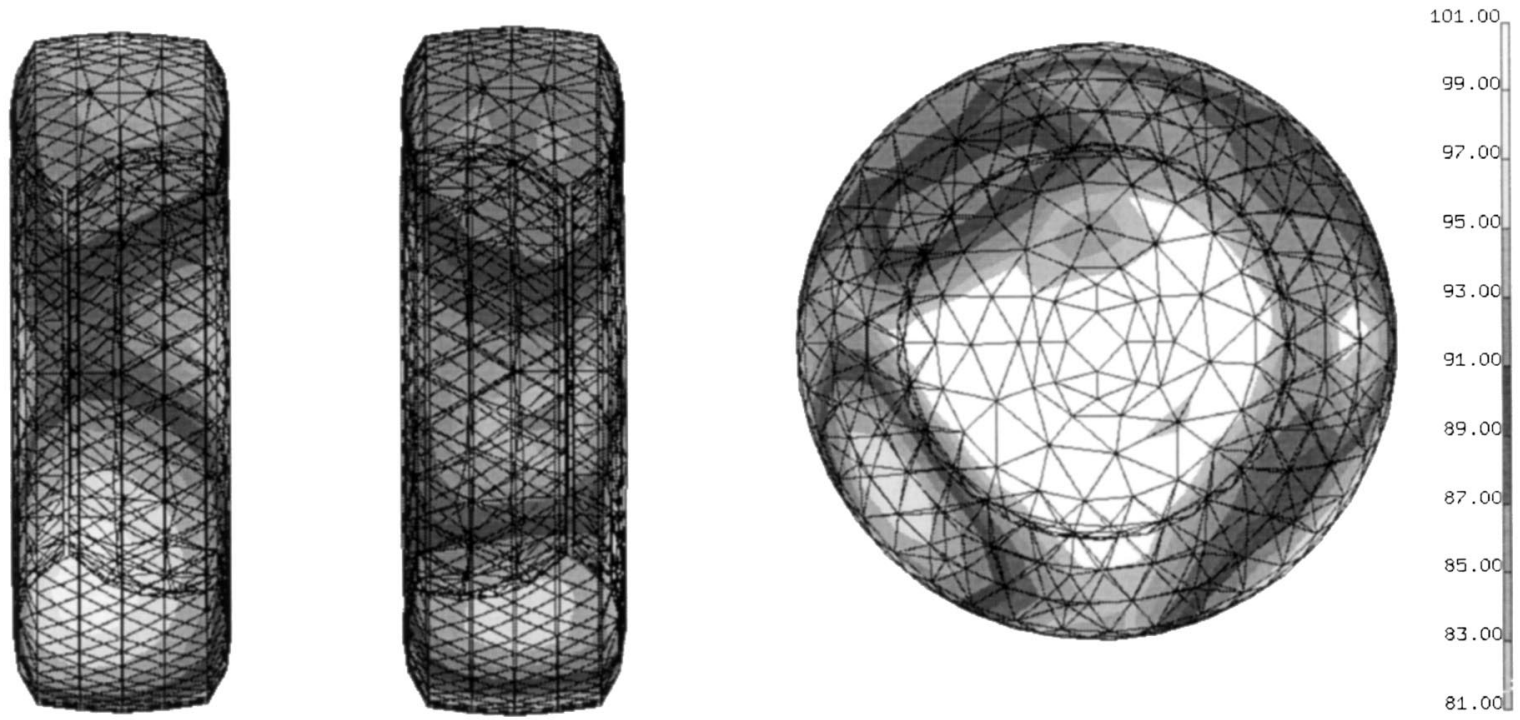

FIG. 5. Reconstructed surface velocity for shaker excited tire at $100 \mathrm{~Hz}$.

tical part of each L-curve and thereby illustrating the differences obtained for this case.

The reason for the success of the L-curve criterion is that it does not rely on the noise being spatially white as GCV does; also colored/correlated noise gives rise to a distinct corner on the curve. The GCV function, on the other hand, may fail to have a suited minimum when the noise is not spatially white.

The computed regularised surface velocity solution for each principal component represents a principal source related to the vibrating tire structure. This total surface velocity distribution is shown on the tire model in Fig. 5. Note that particle velocity
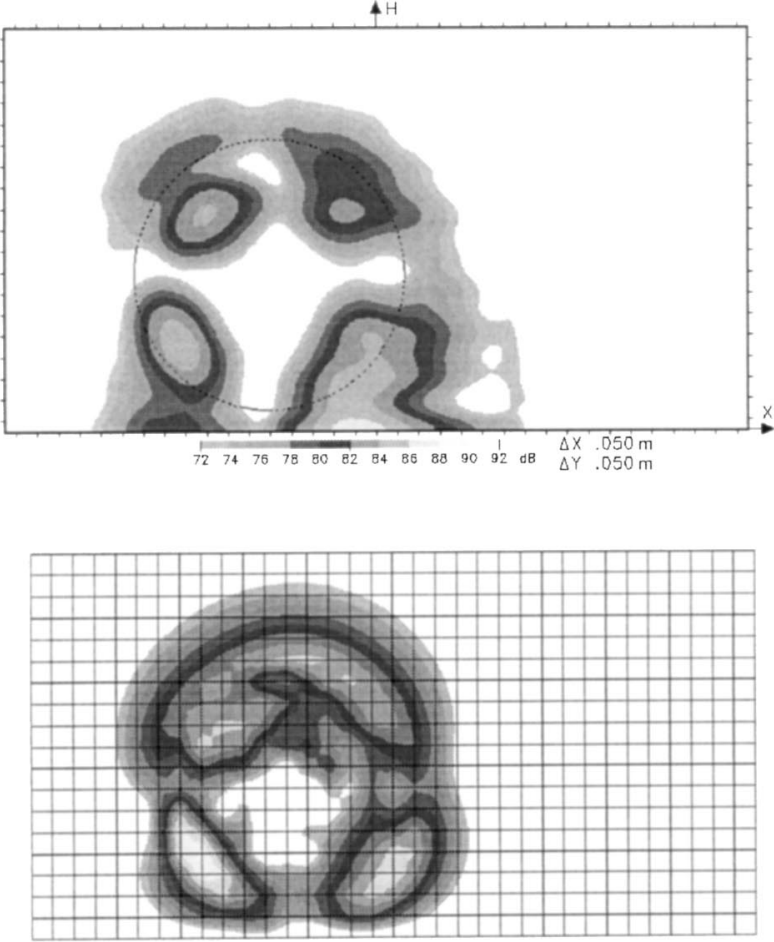

sound pressure
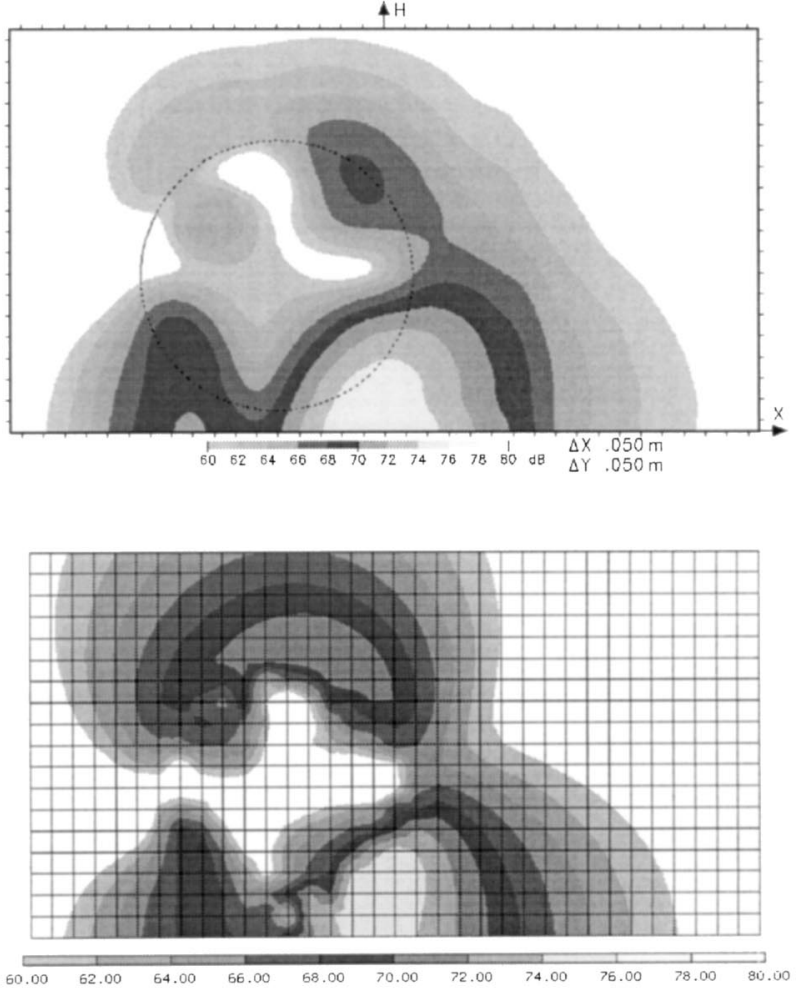

FIG. 6. Reconstructed normal particle velocity and sound pressure on a calculation plane $2 \mathrm{~cm}$ from the tire side of the shaker excited tire at $100 \mathrm{~Hz}$. Results for STSF (top) and IBEM using L-curve criterion (bottom). 

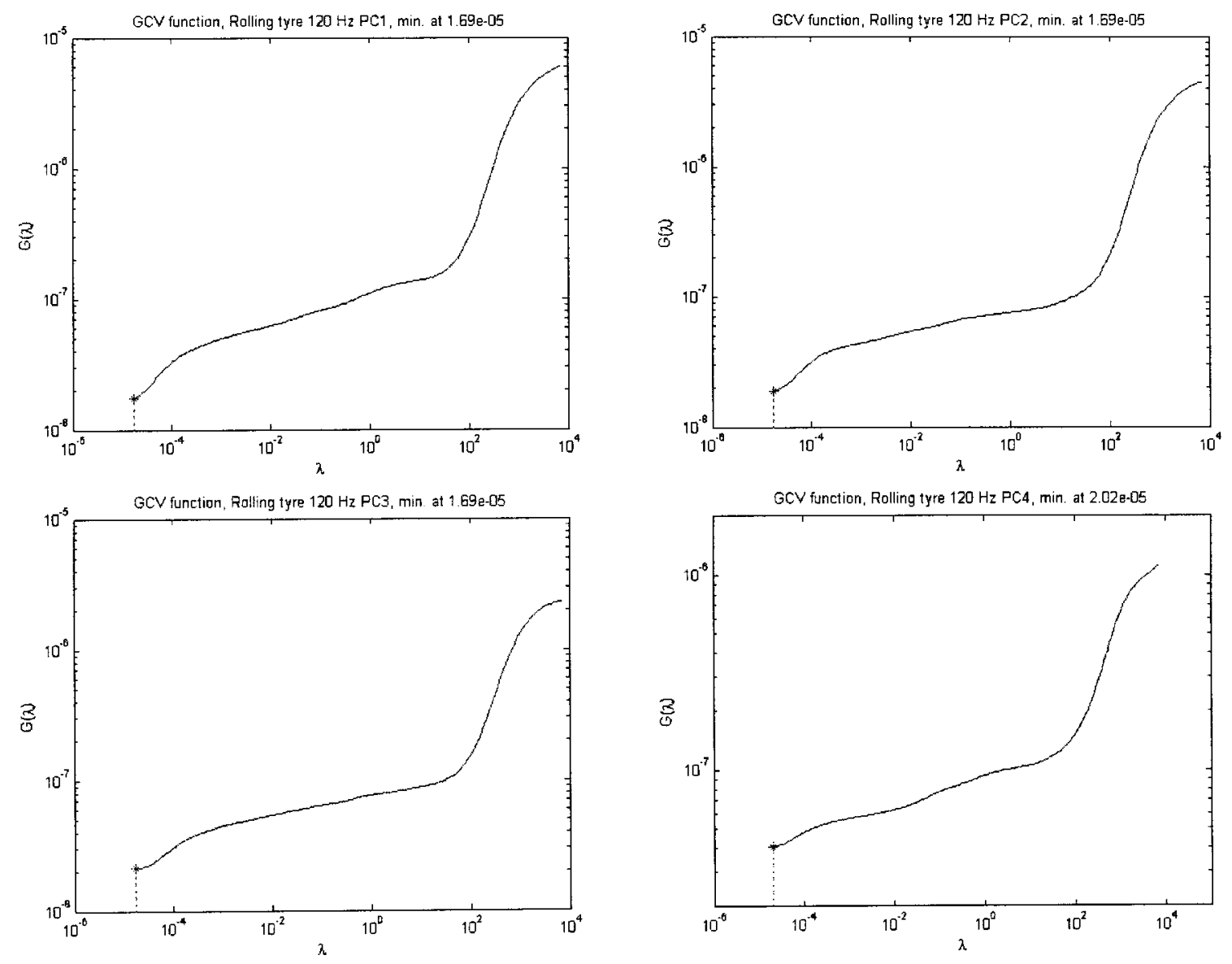

FIG. 7. GCV functions processed for each principal hologram for rolling tire at $120 \mathrm{~Hz}$.

only the highest $20 \mathrm{~dB}$ of the available data are plotted. Since we can observe the tire from any position, we can look at the spot where the shaker excites the tire (front). This reveals a significant hot spot associated with a maximum vibration level. Further sources of vibration are found on the rear part of the tire close to the ground and on the tire sidewall. This surface velocity reconstruction will now be used as boundary condition in a forward BEM problem for computing the sound field close to the tire.

In order to compare the sound field reconstructions obtained by IBEM and STSF, the sound pressure and the particle velocity was computed on a plane close to the tire sidewall. Using STSF, this was done by processing the measurement data from the plane array measurement at the tire side, and then backward propagating the acoustic field $0.05 \mathrm{~m}$ onto a plane close to the tire side. A similar filtering (15 dB dynamic range, smooth cutoff) of the individual principal components was done, but for the regularization part a fixed dynamic range of $30 \mathrm{~dB}$ was used for every principal component. This is simply because no automatic detection of a useful dynamic range is implemented in the current STSF application and $30 \mathrm{~dB}$ is the maximum allowed range. When using IBEM with the L-curve criterion, the principal holograms were processed independently in order to obtain a set of principal sources. These sources were then processed as part of a forward BEM problem for computing the normal component of the particle velocity and the acoustic pressure over the same plane as in the STSF computation. The par- ticle velocity and pressure distributions obtained are shown in Fig. 6 together with the result of the STSF calculation. Both the sound pressure level distributions and the particle velocity distributions obtained by STSF and by IBEM (Lcurve), see Fig. 6, are in good agreement. The levels are almost identical and are shown using the same color bar with the same step size. Clearly, the L-curve criterion applied to each principal component leads to a useful reconstructed particle velocity distribution judged from the STSF calculations. However, it would be more convincing to have the actual measurement data for the calculation plane when doing comparisons.

As mentioned previously, the frequency bandwidth is probably the major reason that we do not see only a single nonzero principal component. Nonzero components arising because of the bandwidth represent real sound fields and real sources. The only error in our IBEM equations will be in the transfer matrix and arise from the fact that we have assumed all sound field components to be at the center frequency of the band. But beyond the frequency bandwidth, there are other causes for a nonzero principal component, which have no relation to real sources. The two components dominated by noise and errors represent a challenge to the applied regularization schemes. From the computed L-curves it is clear that the noisy principal components should be discarded since there is nearly only a vertical part, which can easily lead to very small regularization parameters and thus solutions with a significant norm. For that reason a lower limit on 

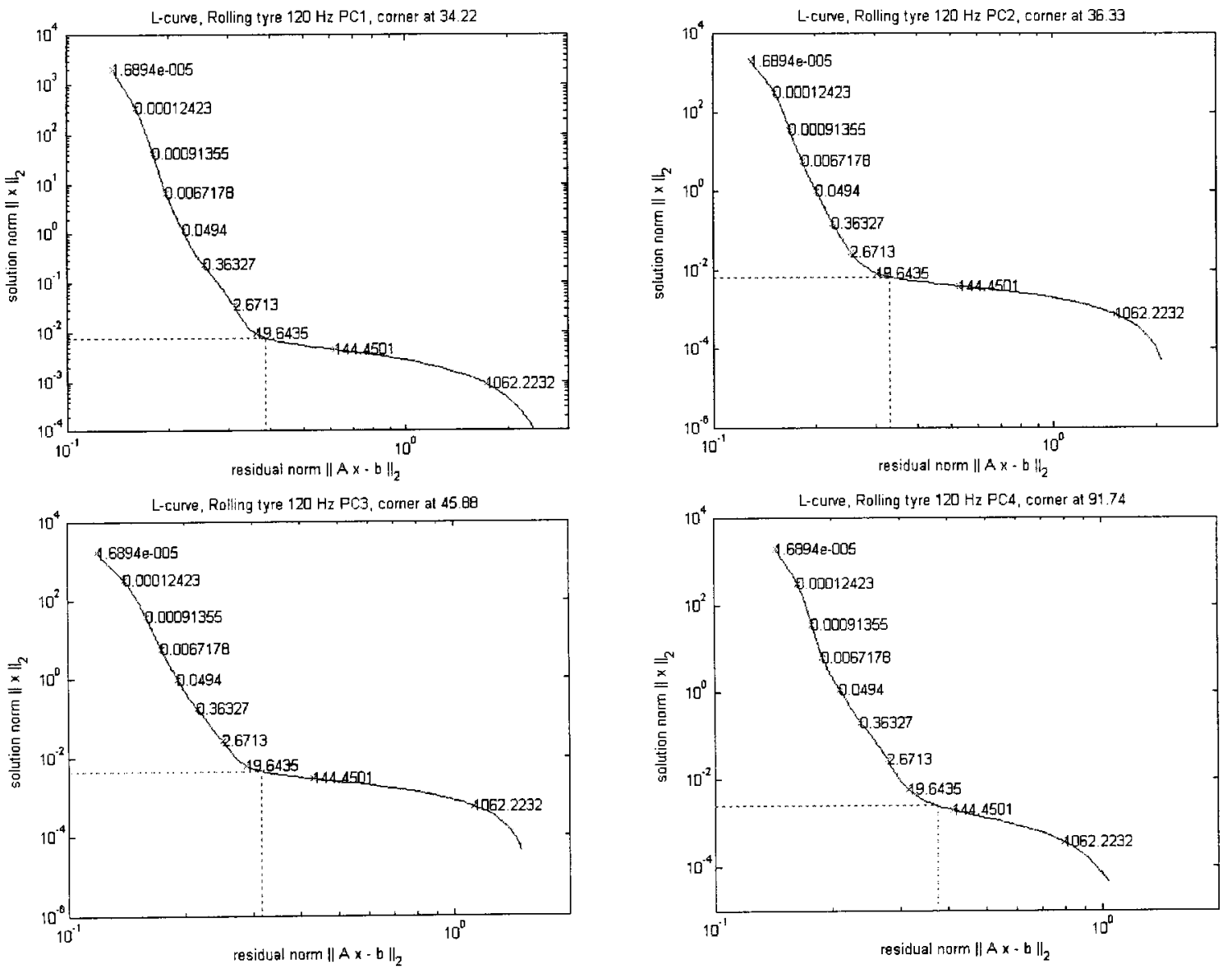

FIG. 8. L-curves corresponding to each principal hologram for rolling tire at $120 \mathrm{~Hz}$. The regularization parameter is marked on the curve.

the regularization parameter could be imposed or a sharp principal component filter should be used. The solutions of Fig. 6 obtained by STSF and by IBEM are dominated by the first principal component with the highest principal autopower.

\section{Rolling tire}

A similar analysis is carried out for the rolling tire case. One major difference between the two measurement cases is that the rolling tire measurement includes a curved array

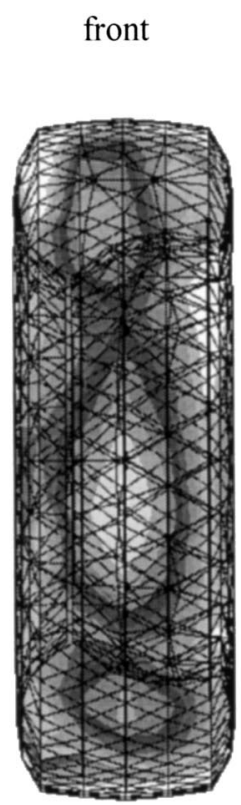

rear

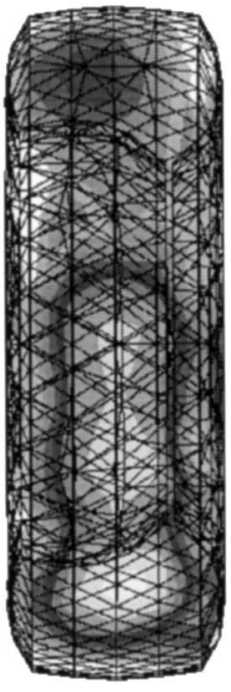

side

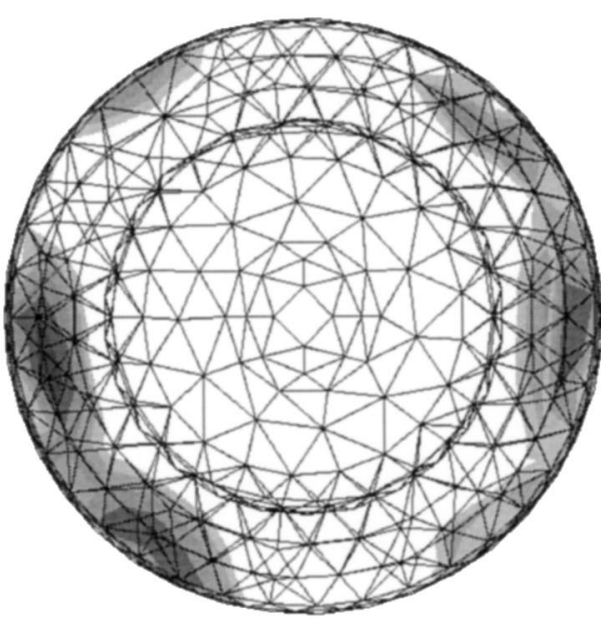

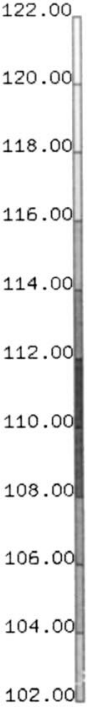

FIG. 9. Reconstructed surface velocity for rolling tire at $120 \mathrm{~Hz}$ 

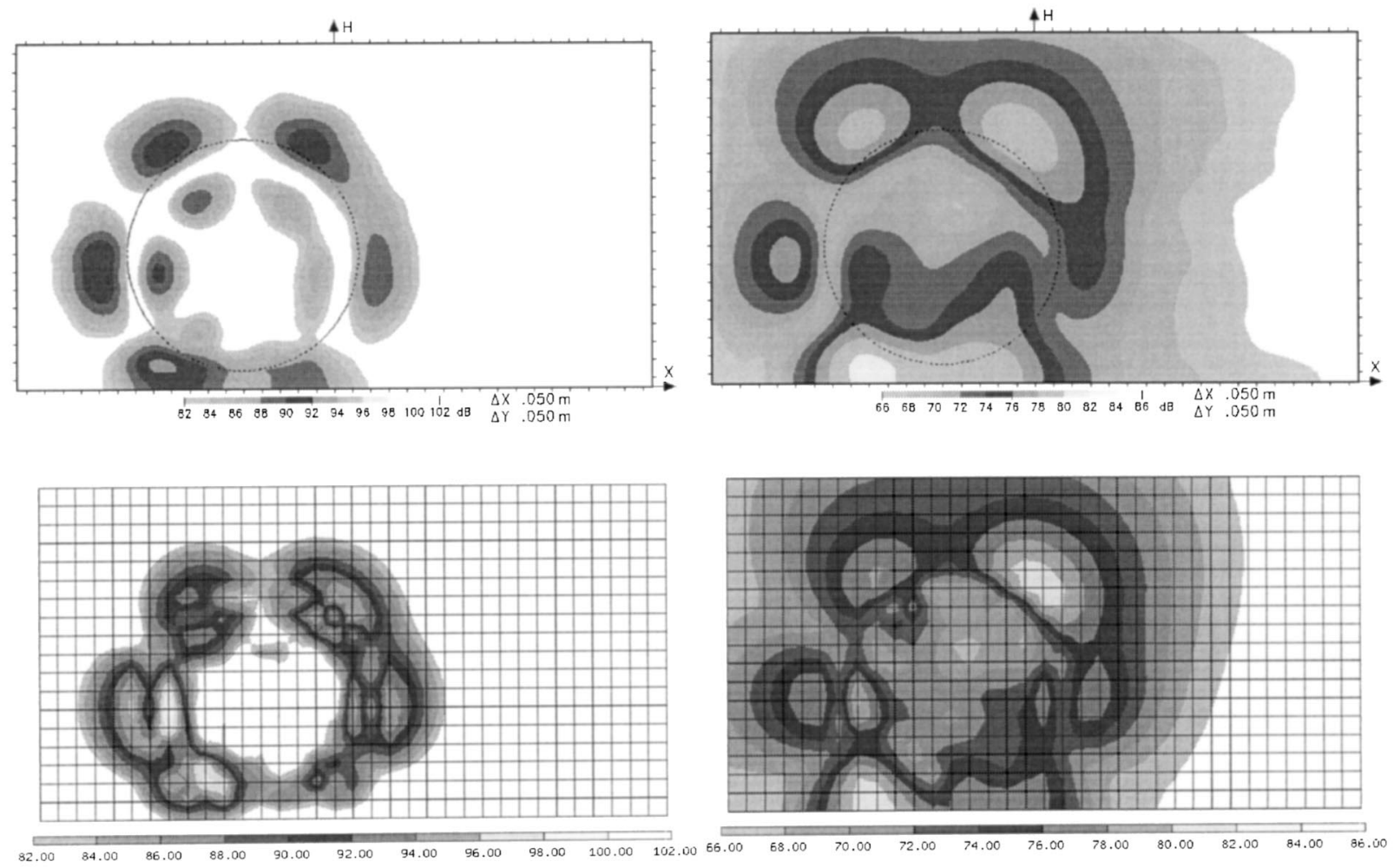

FIG. 10. Reconstructed normal particle velocity and sound pressure on calculation plane $2 \mathrm{~cm}$ from the tire side of the rolling tire at $120 \mathrm{~Hz}$. Results for STSF (top) and IBEM using L-curve criterion (bottom).

scanning of the tire front, thus providing more information about the total sound field around the rolling tire. Again, this curved array was located $0.07 \mathrm{~m}$ from the tire and scanned with a step size of $0.05 \mathrm{~m}$. By adding these front measurement positions to the plane and curved array measurement positions from the shaken tire setup, we end up with a total of 977 measurement points.

Using the same set of four reference microphones, a principal component description of the sound field was derived. Again, we use a low frequency, this time corresponding to a band of width $20 \mathrm{~Hz}$ centered at $120 \mathrm{~Hz}$. For the analysis, this gives us a $977 \times 526$ transfer matrix for the rolling tire. Each of the four principal holograms at $120 \mathrm{~Hz}$ is again associated with an inverse problem. Compared to the shaken tire measurement, we will now for the rolling tire expect some additional errors arising from the rotating drum producing background noise, as well as wind noise induced in the microphones due to wind from the fast rotating tire. However, these additional error components will be rather uncorrelated with the noise radiated from the tire, so provided they are not too large they are not expected to cause major additional problems. A major difference from the case of the shaked tire measurement is that now we will have four significant principal components-all four containing information about the noise due to tire vibration. The autopowers of the second, third, and fourth principal component was 5, 10 , and $15 \mathrm{~dB}$, respectively, below the autopower of the first principal component.

Similar to before, a SVD analysis reveals that the regu- larization parameters for this case should be about 50 . The GCV function for each of the four right-hand sides is shown in Fig. 7 and the computed minimum is marked. Again, the GCV leads to severely undersmoothed solutions, as the estimated regularization parameters are all far too small. It is also noted that the GCV functions in this case start increasing slowly followed by a more rapid increase, which results in minimums on the left part of the curve. This is simply a consequence of the noise and errors in the measurement data and the resulting computed surface velocity distribution will also in this case be completely meaningless.

The L-curve analysis gives the four curves shown in Fig. 8 , where each curve has a distinct corner, even for this complicated case. The vertical part of each curve is not strictly vertical, indicating that the errors are far from being spatially white. For small regularization parameters we see that the residual norm continues to decrease when regularization is turned further down, explaining why the GCV functions behave as in Fig. 7. Furthermore, the computed regularization parameters are all close to 50 in case of the L-curve criterion, which agrees very well with a SVD analysis. The dynamic range of the applied Tikhonov filters is in this case 46, 46, 44 , and $38 \mathrm{~dB}$ for principal component one to four, respectively. The higher dynamic ranges for the rolling tire is because the surface velocity distribution is made up by more SVD components as long as they are not dominated by noise. The difference in solution between the two test cases can be seen from Fourier coefficients; for the shaker excited tire, the 
Fourier coefficients $\left|\mathbf{u}_{i}^{H} \mathbf{p}_{f}\right|$ decay more rapidly than for the rolling tire case until they both hit a noise level.

The total surface velocity distribution on the tire model is shown in Fig. 9, where only the highest $20 \mathrm{~dB}$ of the available data are plotted. From the front and the rear of the tire we find the strongest vibration on the tire tread, especially close to the leading (front) and the trailing edge (rear) of the tire. Further vibration is associated with the tire sidewall.

As with the foregoing case, we compare with results from STSF. The reconstructed surface velocity data are fed into a forward BEM calculation for computing the sound field on the STSF calculation plane. The STSF calculations are again done with a fixed dynamic range of $30 \mathrm{~dB}$ for each principal component. The obtained results for particle velocity and pressure are shown in Fig. 10. In this case, there is a slight difference in level for the particle velocity calculation, which is due to higher dynamic ranges for the IBEM reconstructions. On the other hand, both methods show the same regions of maximum level. For the pressure distribution, we obtain comparable results from the two methods. This is because the pressure distribution does not require as many high spatial frequencies as the particle velocity distribution. The higher dynamic ranges therefore do not have the same impact. These considerations illustrate how useful is the automatic ( $\|\mathbf{e}\|_{2}$-free) detection of the applicable dynamic range.

\section{CONCLUSIONS}

A model of an inverse acoustic source problem was made using the indirect variational BEM formulation described in the first part of the paper. A transfer matrix relating field pressures to surface velocities was formed to take part in a Tikhonov regularization problem. The crucial part associated with choosing the optimal amount of regularization was discussed in the context of error-free parameter-choice methods. The optimal amount of regularization was estimated using the L-curve criterion as well as the popular GCV method for two different tire applications. In both cases we compared reconstructed acoustic field quantities on a plane surface close to the tire side with data from near-field acoustic holography (STSF). In all our experiments, the L-curve criterion provided realistic source reconstructions. The reconstructed source distributions computed by means of GCV were incorrect as a result of the complicated error distributions contaminating the measured data. While the GCV method is well established for reconstruction in the presence of spatially white noise, the cases investigated in this work demonstrate that the L-curve method may be a better choice for practical measurements, where spatially correlated measurement errors such as transducer phase and position errors are present. As a consequence, the L-curve criterion seems to be more robust than the GCV for estimating the optimal amount of regularization.

${ }^{1}$ F. Augusztinovicz and M. Tournour, "Reconstruction of source strength distribution by inversing the boundary element method," in Boundary Elements in Acoustics, edited by O. von Estorff (WIT Press, Southampton, 2000).

${ }^{2}$ M. R. Bai, "Application of BEM (boundary element method)-based acoustic holography to radiation analysis of sound sources with arbitrarily shaped geometries," J. Acoust. Soc. Am. 92, 533-549 (1992).

${ }^{3}$ G.-T. Kim and B.-H. Lee, "3-D sound source reconstruction and field reprediction using the Helmholtz integral equation," J. Acoust. Soc. Am. 136, 245-261 (1990).

${ }^{4}$ W. A. Veronesi and J. D. Maynard, "Digital holographic reconstruction of sources with arbitrarily shaped surfaces," J. Acoust. Soc. Am. 85, 588598 (1989).

${ }^{5}$ Z. Zhang, N. Vlahopoulos, S. T. Raveendra, T. Allen, and K. Y. Zhang, “A computational acoustic field reconstruction process based on an indirect boundary element formulation," J. Acoust. Soc. Am. 108, 2167-2178 (2000).

${ }^{6}$ E. G. Williams, B. H. Houston, P. C. Herdic, S. T. Raveendra, and B. Gardner, "Interior near-field acoustical holography in flight," J. Acoust. Soc. Am. 108, 1451-1463 (2000).

${ }^{7}$ B.-K. Kim and J.-G. Ih, "On the reconstruction of the vibro-acoustic field over the surface enclosing an interior space using the boundary element method," J. Acoust. Soc. Am. 100, 3003-3016 (1996).

${ }^{8}$ A. Schuhmacher, J. Hald, and E.-U. Saemann, "A comparison of inverse boundary element method and near-field acoustical holography applied to sound radiation from a tire," Proceedings of the Sixth International Congress on Sound and Vibration, Copenhagen, 1999.

${ }^{9}$ A. Schuhmacher, "Practical application of inverse boundary element method to sound field studies of tires,' Proceedings of Internoise '99, Ft. Lauderdale, 1999.

${ }^{10}$ B.-K. Kim and J.-G. Ih, "Design of an optimal wave-vector filter for enhancing the resolution of reconstructed source field by near-field acoustical holography (NAH)," J. Acoust. Soc. Am. 107, 3289-3297 (2000).

${ }^{11}$ P. A. Nelson, "Some inverse problems in acoustics," in Ref. 8.

${ }^{12}$ P. A. Nelson and S. H. Yoon, "Estimation of acoustic source strength by inverse methods: Part II: Methods for choosing regularisation parameters," ISVR Technical Report No. 279, University of Southampton, 1998.

${ }^{13}$ E. G. Williams, "Regularization methods for near-field acoustical holography," J. Acoust. Soc. Am. 110, 1976-1988 (2001).

${ }^{14}$ P. C. Hansen, "Analysis of discrete ill-posed problems by means of the L-curve," SIAM Rev. 34, 561-580 (1992).

${ }^{15}$ R. Martinez, "The thin-shape breakdown (TSB) of the Helmholtz integral equation," J. Acoust. Soc. Am. 90, 2728-2738 (1991).

${ }^{16}$ V. Cutanda, P. M. Juhl, and F. Jacobsen, "On the modeling of narrow gaps using the standard BEM,” J. Acoust. Soc. Am. 109, 1296-1303 (2001).

${ }^{17}$ P. J. T. Filippi, "Layer potentials and acoustic diffraction," J. Sound Vib. 54, 473-500 (1977).

${ }^{18}$ M. N. Sayhi, Y. Ousset, and G. Verchery, "Solution of radiation problems by collocation of integral formulations in terms of single and double layer potentials," J. Sound Vib. 74, 187-204 (1981).

${ }^{19}$ X. Zeng, J. Bielak, and R. C. MacCamy, "Unified symmetric finite element and boundary integral variational coupling methods for linear fluidstructure interaction," Numerical Methods for Partial Differential Equations 8, 451-467 (1992).

${ }^{20}$ O. von Estorff, A. Homm, and F. Bartels, "Sound radiation of a cantilever plate-comparisons of BEM results with experimental data," Betech 91, Southampton, 1991.

${ }^{21}$ O. von Estorff, M. Fischer, and W. J. M. Keiper, "Sound radiation of a tuning-fork by boundary elements," BEM 14, Seville, 1992.

${ }^{22}$ S. T. Raveendra, N. Vlahopoulos, and A. Glaves, “An indirect boundary element formulation for multi-valued impedance simulation in structural acoustics," Appl. Math. Model. 22, 379-393 (1998).

${ }^{23}$ R. Jeans and I. C. Mathews, "A comparison of numerical collocation and variational procedures to the hypersingular acoustic integral operator," Comput. Methods Appl. Mech. Eng. 101, 5-26 (1992).

${ }^{24}$ S. T. Raveendra, "Analysis of periodic acoustic problems with double sided impedance condition," Proceedings Noise-Con '97, Pennsylvania, 1997.

${ }^{25}$ G. H. Golub and C. F. van Loan, Matrix Computations (The Johns Hopkins University Press, Baltimore, MD, 1996).

${ }^{26}$ P. C. Hansen, M. E. Kilmer, and R. H. Kjeldsen, "Exploiting residual information in the regularisation of discrete ill-posed problems," SIAM J. Sci. Comput. (USA) (submitted).

${ }^{27}$ P. C. Hansen, Rank-Deficient and Discrete Ill-Posed Problems (SIAM, Philadelphia, 1998).

${ }^{28}$ P. C. Hansen and D. P. O'Leary, "The use of the L-curve in the regularisation of discrete ill-posed problems," SIAM J. Sci. Comput. (USA) 14, 1487-1503 (1993). 
${ }^{29}$ A. P. Schuhmacher, "Sound source reconstruction using inverse sound field calculations," Ph.D. thesis, Department of Acoustic Technology, Technical University of Denmark, Report 77, 2000.

${ }^{30}$ S.-C. Kang and J.-G. Ih, "On the accuracy of nearfield pressure predicted by the acoustic boundary element method," J. Sound Vib. 233, 353-358 (2000).

${ }^{31}$ S.-C. Kang and J.-G. Ih, "Use of nonsingular boundary integral formulation for reducing errors due to near-field measurements in the boundary element method based near-field acoustic holography," J. Acoust. Soc. Am. 109, 1320-1328 (2001).

${ }^{32}$ Z. Zhang, N. Vlahopoulos, T. Allen, and K. Y. Zhang, "A source recon- struction process based on an indirect variational boundary element formulation," Eng. Anal. Boundary Elem. 25, 93-114 (2001).

${ }^{33}$ H.-S. Kwon, J. S. Bolton, and D. L. Hallman, "Nearfield acoustical holography applied to sound radiation from tires," Proceedings Noise-Con '98, Ypsilanti, 1998.

${ }^{34}$ J. Hald, "STSF - a unique technique for scan-based near-field acoustic holography without restrictions on coherence," Brüel \& Kjær Technical Review No. 1, 1989.

${ }^{35} \mathrm{~J}$. Hald, "STSF-a unique technique for scan-based near-field acoustic holography without restrictions on coherence," Brüel \& Kjær Technical Review No. 2, 1989. 